\title{
Differential Changes in Dietary Habits after Gastric Bypass Versus Gastric Banding Operations
}

\author{
Barbara Ernst • Martin Thurnheer • Britta Wilms • \\ Bernd Schultes
}

Received: 15 August 2008 / Accepted: 4 November 2008 / Published online: 26 November 2008

(C) Springer Science + Business Media, LLC 2008

\begin{abstract}
Background Reduction of food intake is an important mechanism by which bariatric procedures reduce body weight. However, only few studies have systematically assessed what patients actually eat after different types of bariatric operations.

Methods Dietary habits were assessed by a food frequency questionnaire in 121 bariatric patients (48 gastric bypass patients, 73 gastric banding patients) during follow-up visits in our interdisciplinary obesity center as well as in 45 severely obese (body mass index $(\mathrm{BMI})>35 \mathrm{~kg} / \mathrm{m}^{2}$ ) and 45 nonobese $\left(\mathrm{BMI}<27 \mathrm{~kg} / \mathrm{m}^{2}\right)$ control subjects.

Results As compared with nonobese control subjects, obese control subjects consumed more meat, white bread, and diet soft drinks. Gastric bypass patients showed an enhanced consumption of foods rich in protein such as poultry, fish, and eggs as well as of cooked vegetables, while the consumption of fatty sweets like chocolate, cake, biscuits, and cookies was found to be distinctly reduced in this patient group. In contrast, gastric banding patients reported on a reduced intake of pasta, white bread, and fresh fruits and, just like gastric bypass patients, also on an enhanced intake of poultry and fish. Direct comparison of dietary habits between the two bariatric patient groups revealed that
\end{abstract}

The authors have no commercial interest to disclose. The study was financially supported by a grant from Johnson \& Johnson. The funder had no role in the study design, analysis, and publication of the data.

B. Ernst $\cdot$ M. Thurnheer $\cdot$ B. Schultes $(\triangle)$

Interdisciplinary Obesity Center,

Kantonsspital St. Gallen,

9400 Rorschach, Switzerland

e-mail: bernd.schultes@kssg.ch

B. Wilms • B. Schultes

Department of Internal Medicine I, University of Luebeck,

23538 Lübeck, Germany gastric bypass patients consumed more frequently fresh fruits, eggs, and diet soft drinks but strikingly less chocolate than gastric banding patients.

Conclusion Collectively, data clearly point to distinct changes in dietary habits after bariatric operations which markedly differ between gastric bypass and gastric banding patients. Overall, it is tempting to conclude that gastric bypass operations lead to a healthier and a more balanced diet than gastric band implantations.

Keywords Food selection - Avoiding behavior . Dietary counseling $\cdot$ Food frequency questionnaire . Morbid obesity

\section{Introduction}

Reduction of food intake represents the main effect by which purely restrictive bariatric procedures such as gastric banding decreases body weight, but it also plays an important role in gastric bypass procedures [1]. Depending on the specific kind of bariatric operation, the causes for reduced food intake can rely on distinctly different mechanisms. Firstly, the narrow passage across the gastric band or across the gastric anastomosis after gastric bypass operations can limit the consumption of several types of foods that cannot be chewed very well into small pieces, e.g., foods with a high amount of insoluble fibers. Secondly, gastric bypass operation can limit the ingestion of simple carbohydrates since the consumption of such foods can be followed by the unpleasant symptoms of a dumping syndrome $[2,3]$. Also, fatty foods may provoke adverse reactions like fatty stools and diarrhea [2, 3]. Lastly, many patients report on changes in feelings of appetite and hunger after bariatric operations which may 
derive from their distinct influences on the secretion of gastrointestinal hormones that are critically involved in the regulation of appetite and eating behavior [4].

Despite these profound influences of bariatric surgery on food intake, only a limited number of studies have systematically assessed what patients actually eat after different bariatric operations. Of those, most studies have focused on the quantitative intake of energy [5-11], macronutrient components [5-8, 11-13], or specific micronutrients such as calcium [6, 7, 9, 10, 14] while only few studies have determined the ingestion of specific types of food such as meat, fruits, vegetables, sweets, etc. [15-18]. Shai et al. [15] reported on a reduced intake of meat, fish, vegetables, bread, eggs, sweets, and water after vertical banded gastroplasty, while the intake of dairy products was increased. After gastric bypass operations, a reduced intake of red meat, fish, chicken, turkey, fruits, vegetables, bread, cereals, nuts, milk, cheese, sweet desserts, and soda was observed in another study [16]. However, only one study [17] directly compared the intake of specific foods between vertical banded gastroplasty and gastric bypass patients and found a lower intake of milk and ice cream, high-caloric liquids, and nonliquid sweets after gastric bypass than after vertical banded gastroplasty. Results of another study [18] comparing dietary habits after horizontal gastroplasty vs. gastric bypass, showed in a similar direction with a marked reduction in milk, ice cream, sweets, and high-caloric beverages consumption after the gastric bypass operation but not after the purely restrictive procedure, i.e., the horizontal gastroplasty. However, the interpretation of all these findings is limited by the fact that none of these studies included an untreated obese or a nonobese control group so that it remains unclear how the found dietary habits in bariatric patients compare to that of nonbariatric subjects. Furthermore, while dietary habits after gastroplasty have previously been investigated [15, 17, 18], dietary habits after adjustable gastric band implantation, which is nowadays the most commonly performed purely restrictive procedure [19], have to the best of our knowledge not been reported so far. On this background, here, we assessed dietary habits in patients who have underwent a gastric bypass or gastric banding operation and compared their data with those of an obese as well as a nonobese control group.

\section{Methods}

In this cross-sectional study, we assessed dietary habits in regard of the selection of different foods by a food frequency questionnaire (FFQ) in 121 bariatric patients who routinely came to our follow-up program in the Interdisciplinary Obesity Center (IOC) between September
2007 and May 2008. All patients taking drugs known to influence food intake, e.g., psychotropic drugs, were excluded from the study. Also, patients with a duration of less than 1 year after the operation were excluded. The bariatric sample included 48 patients who had undergone a gastric bypass operation and 73 patients after a gastric band implantation. Of the gastric bypass patients, 18 had a standard proximal gastric bypass, while the remaining 30 patients had a distal gastric bypass with the length of the common channel ranging between 60 and $100 \mathrm{~cm}$. As an obese control group, we applied the FFQ on 45 consecutive severely obese (body mass index $(\mathrm{BMI})>35 \mathrm{~kg} / \mathrm{m}^{2}$ ) subjects who attended our IOC for evaluation of bariatric surgery or other weight loss therapies. In addition, 45 nonobese (BMI 18 to $<27 \mathrm{~kg} / \mathrm{m}^{2}$ ) subjects filled in the FFQ and served as another control group. Written informed consent was obtained from all subjects.

Prior to and after the bariatric operation, all patients received dietary counseling on a regular basis to learn how to adjust their eating behavior to the postoperative situation. Common advices for all types of operations were: small portion sizes (half a cup, three to five tablespoons), eating slowly and chewing very carefully, avoid drinking liquids during meals, and three main meals a day. In regard of food selection, patients were instructed to ensure an adequate protein intake, to avoid fatty foods and sweets, and to preferentially consume sugar-free beverages. In addition, gastric banding patients were advised to avoid beverages with carbonic acid and to prefer foods low in fiber. In case of gastric bypass operations, patients were advised to reduce the consumption of bloating vegetables and fruits.

Dietary habits were assessed by using a standard FFQ composing 24 different food categories. Subjects had to rate on the frequency of intake for each food category on a sixlevel scale: 1 = almost every day, 2 = several times a week, $3=$ once a week, $4=$ several times a month, $5=$ once a month or less frequent, $6=$ never.

Height and weight were measured with patients wearing light clothing but no shoes. BMI was defined as weight $(\mathrm{kg})$ divided by height squared $\left(\mathrm{m}^{2}\right)$. Percent of excess weight loss (\% EWL) was determined by ((preoperative weight-current weight $) /($ preoperative weight - normal weight $) \times 100$ and percent of excess BMI loss ( $\%$ EBL) was determined by ((preoperative BMI-current BMI)/(preoperative BMI25) $) \times 100$.

Data were analyzed by using SPSS 12.0 for Windows (SPSS Inc., Chicago, IL, USA). Unless otherwise indicated, data are reported as mean \pm SE. Differences between groups were assessed by analysis of variance for continuous variables or $\chi^{2}$ test for discrete variables. For pairwise comparisons, unpaired Student's $t$ test was used. A $P$ value $<0.05$ was considered significant. 


\section{Results}

The characteristics of the study population are presented in Table 1. Sex was equally distributed across the four different groups $(P=0.88)$. Also, age did not significantly differ between groups $(P=0.21)$. By selection, the obese control group had a higher BMI than the nonobese control group $(P<0.001)$. The gastric bypass patients had lost on average $81.7 \pm 2.6 \%$ EBL. They were still heavier than the nonobese control group $(P<0.001)$ but significantly lighter than the obese control group $(P<0.001)$. Weight loss was significantly less pronounced in the gastric banding group $(51.0 \pm 2.7 \%$ EBL $)$ than in the gastric bypass group $(P<$ $0.001)$. However, the gastric banding patients were also significantly lighter than the obese control subjects $(P<$ $0.001)$ but heavier than the nonobese control subjects $(P<$ 0.001). Importantly, while the BMI before the bariatric operation was higher in the gastric bypass than gastric banding patients $(P=0.032)$, the preoperative BMI of both the gastric bypass group as well as the gastric banding group did not significantly differ from the BMI of the obese control group ( $P=0.62$ and $P=0.20$; respectively). Of note, the time interval between the current assessment and the bariatric operation was significantly longer in the gastric banding group than in the gastric bypass group (78.9 \pm 3.2 vs. $22.7 \pm 2.3$ months; $P<0.001)$.

Analyses of the FFQ revealed that the obese control subjects consumed significantly more often meat $(2.2 \pm 0.2$ vs. $2.7 \pm 0.2 ; \mathrm{P}=0.046)$, white bread and toast $(2.4 \pm 0.3$ vs. $3.2 \pm 0.3 ; P=0.027)$, and diet soft drinks ( $3.8 \pm 0.3$ vs. $5.3 \pm$ $0.2 ; P<0.001$ ) than the nonobese control subjects (Fig. 1).

Comparison of the 18 patients having a proximal gastric bypass with those having a distal gastric bypass revealed that food selection only differed in regard of a higher intake of sausage products and ham in the distal than proximal gastric bypass group (2.6 \pm 1.2 vs. $3.4 \pm 1.6 ; P=0.046)$. Thus, data of the proximal and distal gastric bypass patients were pooled for further analyses.
As compared with the nonobese control group, the gastric bypass patients showed a more frequent intake of meat $(2.2 \pm 0.1$ vs. $2.7 \pm 0.2 ; P=0.01)$, poultry $(2.8 \pm 0.2$ vs. $3.4 \pm 0.2 ; P=0.014)$, cooked vegetables $(1.7 \pm 0.1$ vs. $2.1 \pm$ $0.1 ; P=0.002)$, eggs $(2.7 \pm 0.2$ vs. $3.3 \pm 0.2 ; P=0.007)$, and diet soft drinks $(3.3 \pm 0.3$ vs. $5.3 \pm 0.2 ; P<0.001)$, while they consumed less chocolate $(3.7 \pm 0.2$ vs. $2.8 \pm 0.2 ; P=0.01)$, cake, biscuits and cookies $(3.7 \pm 0.2$ vs. $3.0 \pm 0.2 ; P=0.015)$, and cereals ( $4.1 \pm 0.2$ vs. $3.0 \pm 0.3 ; P=0.003$; Fig. $2 \mathrm{a}$ ). As compared with the obese control subjects, gastric bypass patients consumed more often poultry $(2.8 \pm 0.2$ vs. $3.7 \pm$ $0.2 ; P=0.002)$, fish $(3.7 \pm 0.2$ vs. $4.3 \pm 0.2 ; P=0.034)$, cooked vegetables $(0.17 \pm 0.1$ vs. $2.2 \pm 0.2 ; P=0.005)$, and eggs $(2.7 \pm 0.2$ vs. $3.4 \pm 0.2 ; P=0.004)$, while they consumed less chocolate $(3.7 \pm 0.2$ vs. $2.8 \pm 0.2 ; P=0.014)$, cake, biscuits and cookies ( $3.7 \pm 0.2$ vs. $3.1 \pm 0.2 ; P=0.046)$, and soda ( $1.9 \pm 0.3$ vs. $1.3 \pm 0.2 ; P=0.035$; Fig. $2 b)$. A trend was also noted for a less frequent consumption of white bread and toast $(3.1 \pm 0.2$ vs. $2.4 \pm 0.2 ; P=0.06)$ and whole-grain bread $(2.7 \pm 0.2$ vs. $2.2 \pm 0.2 ; P=0.06)$ in the gastric bypass patients.

Gastric banding patients consumed more frequently poultry $(2.9 \pm 0.1$ vs. $3.4 \pm 0.2 ; P=0.017)$ and diet soft drinks $(4.1 \pm 0.2$ vs. $5.3 \pm 0.2 ; P<0.001)$ than the nonobese control subjects while they consumed less frequently fresh fruits $(2.5 \pm 0.2$ vs. $1.4 \pm 0.2 ; P<0.001)$ and fruit juice and soft drinks (3.8 \pm 0.2 vs. $2.9 \pm 0.3 ; P=0.018$; Fig. 3a). Furthermore, a trend was noted for a more frequent consumption of meat $(2.3 \pm 0.2$ vs. $2.7 \pm 0.2 ; P=0.06)$ and fish $(3.6 \pm 0.1$ vs. $4.0 \pm 0.2 ; P=0.06)$ and a less frequent consumption of pasta $(2.9 \pm 0.3$ vs. $2.6 \pm 0.1 ; P=0.08)$ in gastric banding patients. As compared with obese control subjects, gastric banding patients consumed more often poultry $(2.9 \pm 0.1$ vs. $3.7 \pm 0.2 ; P=0.002)$ and fish $(3.6 \pm 0.1$ vs. $4.3 \pm 0.2 ; P=$ $0.005)$ but less often pasta $(2.9 \pm 0.1$ vs. $2.5 \pm 0.2 ; P=0.032)$, fresh fruits $(2.5 \pm 0.2$ vs. $2.0 \pm 0.2 ; P=0.042)$, and white bread and toast $(3.3 \pm 0.2$ vs. $2.4 \pm 0.2 ; P=0.002)$. Also, gastric banding patients tended to consume more frequently

Table 1 Characteristics of the study population

\begin{tabular}{lllll}
\hline & $\begin{array}{l}\text { Nonobese control subjects } \\
n=45\end{array}$ & $\begin{array}{l}\text { Obese control subjects } \\
n=45\end{array}$ & $\begin{array}{l}\text { Gastric bypass patients } \\
n=48\end{array}$ & $\begin{array}{l}\text { Gastric banding patients } \\
n=73\end{array}$ \\
\hline Female/male & $34 / 11$ & $35 / 10$ & $37 / 11$ & $59 / 14$ \\
Age (years) & $39.7 \pm 2.3$ & $42.6 \pm 2.0$ & $40.2 \pm 1.5$ & $44.0 \pm 1.2$ \\
Current BMI $\left(\mathrm{kg} / \mathrm{m}^{2}\right)$ & $22.1 \pm 0.3$ & $45.4 \pm 1.0^{* *}$ & $29.4 \pm 0.7 * *, * * *$ & $34.7 \pm 0.7 * *, * * *, *$ \\
Pre OP BMI $\left(\mathrm{kg} / \mathrm{m}^{2}\right)$ & - & - & $46.5 \pm 0.7$ & $44.6 \pm 0.5^{* * * *}$ \\
$\%$ EWL & - & - & $76.3 \pm 2.4$ & $48.1 \pm 2.6^{*}$ \\
$\%$ EBL & - & - & $81.7 \pm 2.6$ & $51.0 \pm 2.7^{*}$ \\
\hline
\end{tabular}

Data are mean $\pm \mathrm{SE}$ and range. $P$ values are derived from unpaired Student's $t$ test

$* P<0.001$ vs. gastric bypass patients; $* * P<0.001$ vs. nonobese control subjects; $* * * P<0.001$ vs. obese control subjects; $* * * * P<0.05$ vs. gastric bypass patients 
Fig. 1 Comparison of dietary habits between obese (black circle) and nonobese (open circle) control subjects.

${ }^{(*)} P<0.1, * P<0.05$, $* * * P<0.001$

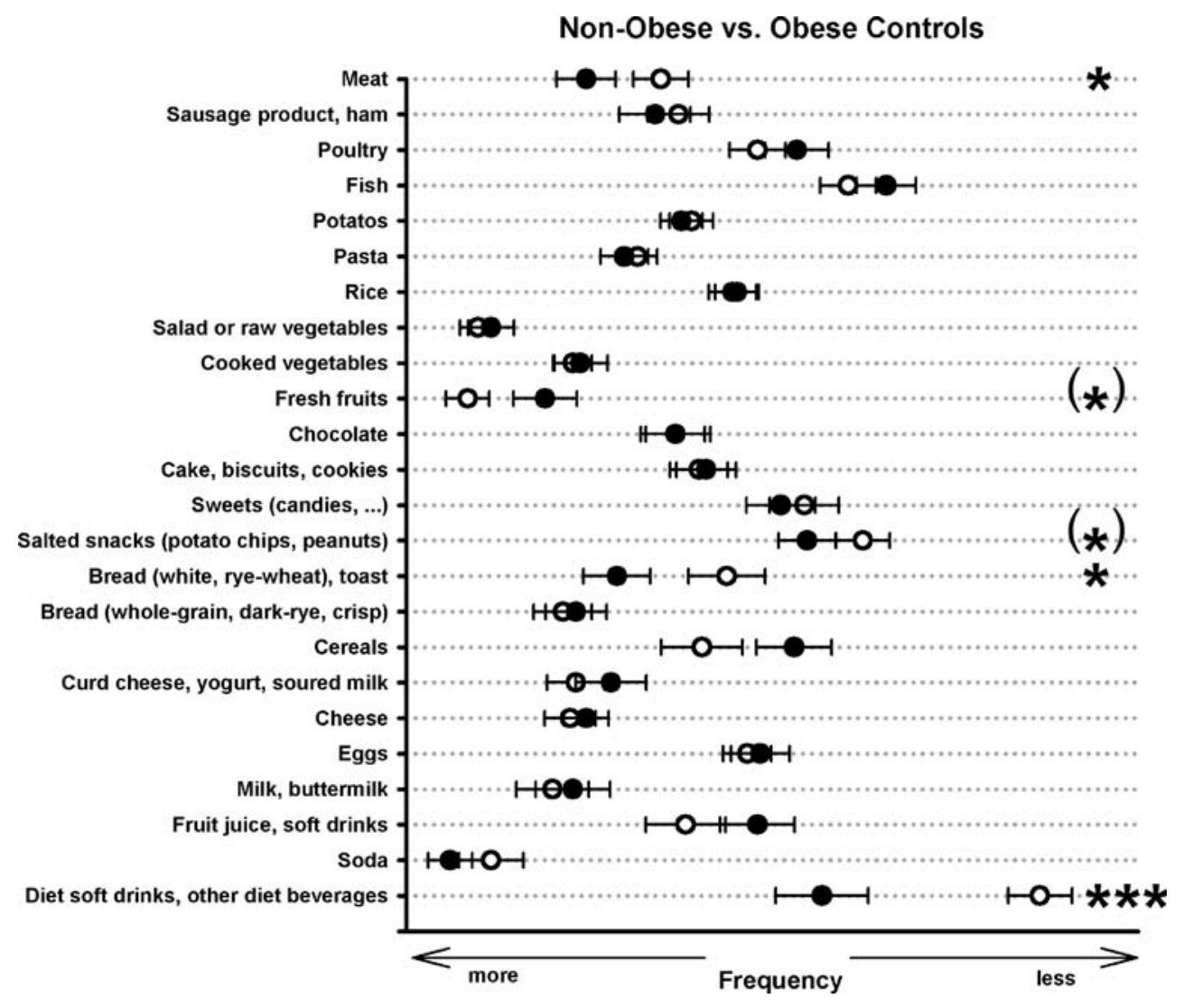

0.2 vs. $3.3 \pm 0.1 ; P=0.004)$, and diet soft drinks $(3.3 \pm 0.3$ vs. $4.1 \pm 0.2 ; P=0.045)$, while they consumed strikingly less chocolate $(3.7 \pm 0.2$ vs. $2.9 \pm 0.2 ; P=0.003)$ than patients after gastric banding. There was also a trend for gastric bypass patients drinking less soda $(1.9 \pm 0.3$ vs. $4.1 \pm 0.2$; $P=0.06$; Fig. 4). a

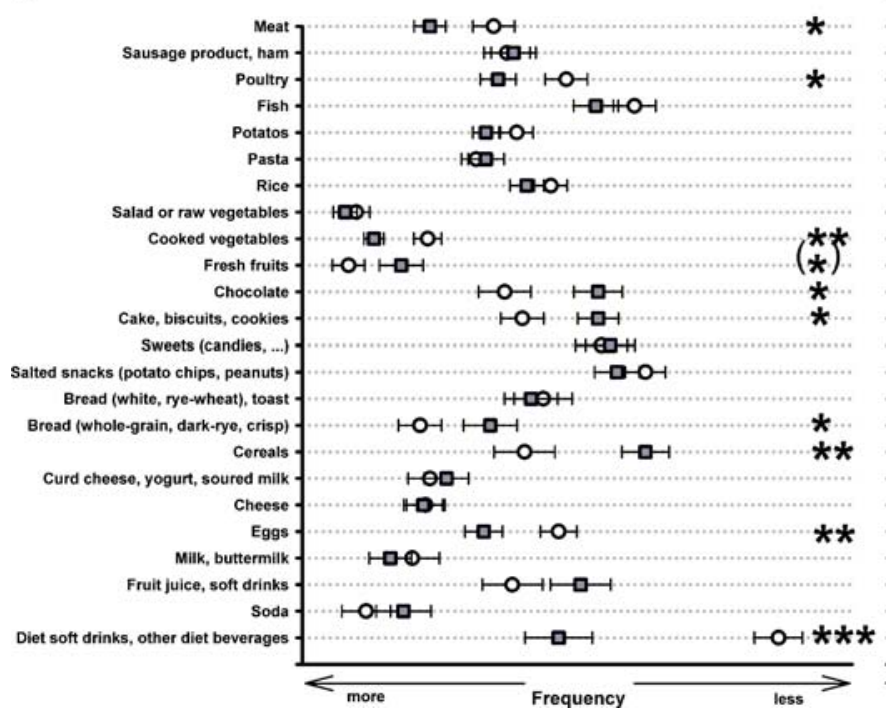

b

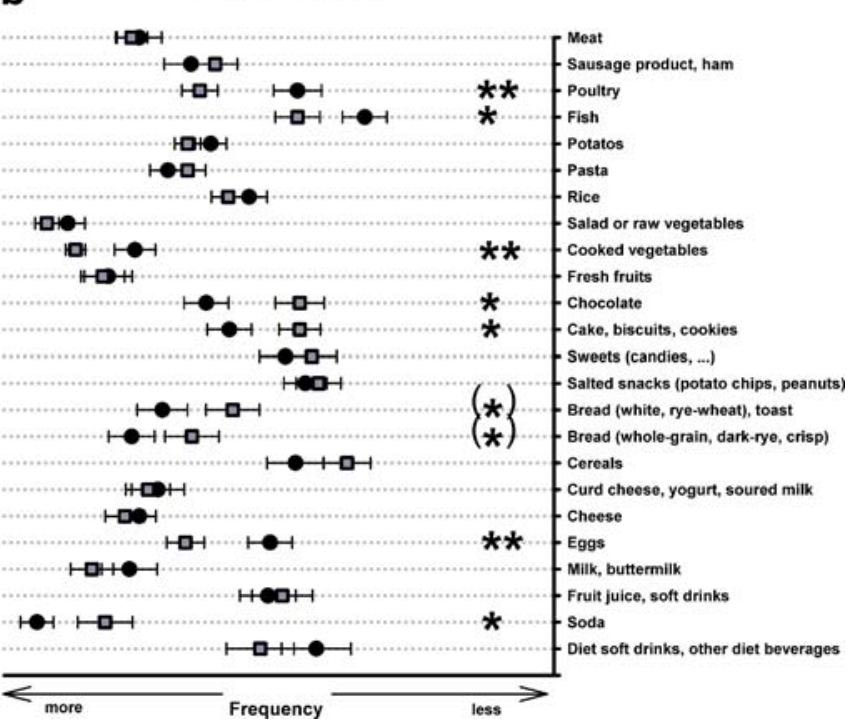

Fig. 2 Comparison of dietary habits between gastric bypass patients (gray squares) and nonobese (open circle) control subjects (a) and between gastric bypass patients and obese (black circle) control subjects (b). ${ }^{(*)} P<0.1, * P<0.05, * * P<0.01, * * * P<0.001$ 

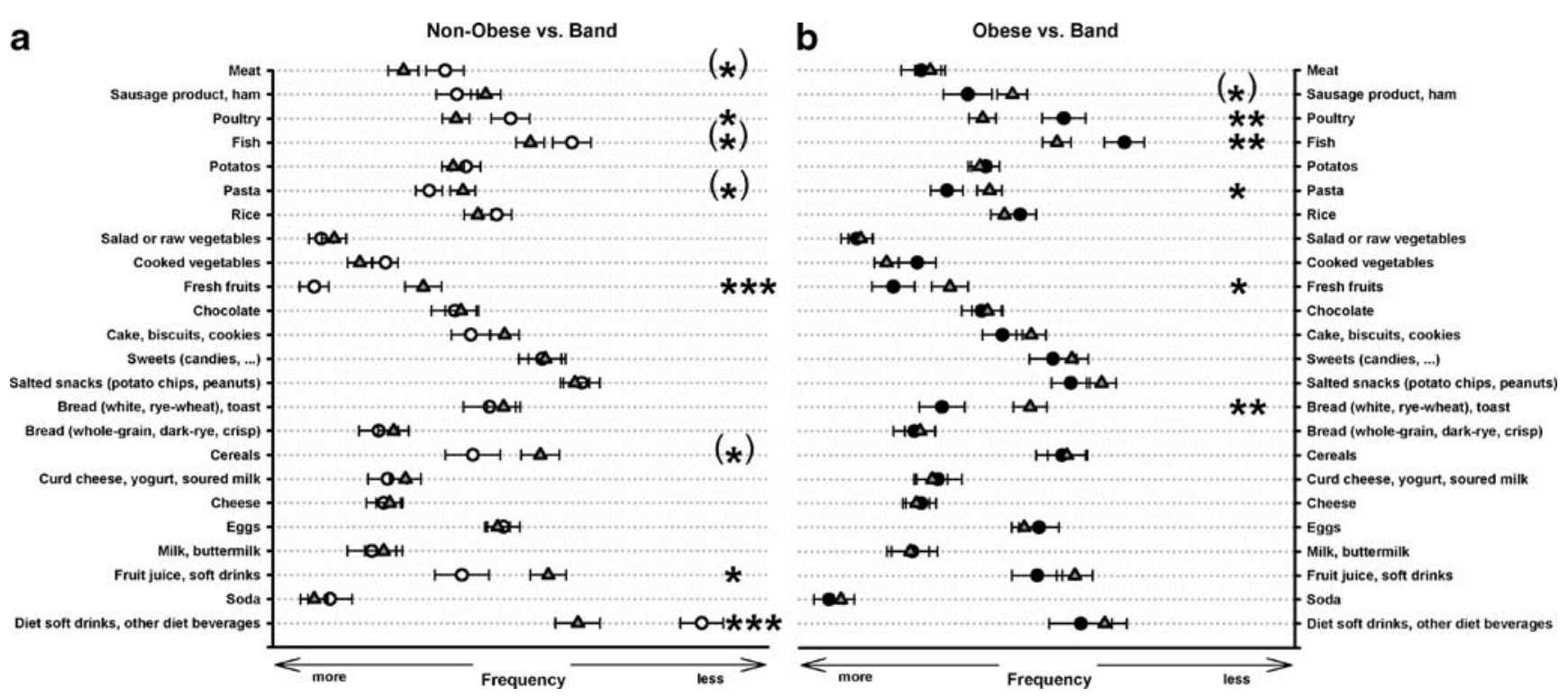

Fig. 3 Comparison of dietary habits between gastric banding patients (gray triangle) and nonobese (open circle) control subjects (a) and between gastric banding patients and obese (black circle) control subjects (b). ${ }^{(*)} P<0.1, * P<0.05,{ }^{* *} P<0.01, * * * P<0.001$

\section{Discussion}

In this cross-sectional study, we assessed dietary habits of obese patients after gastric bypass and gastric banding operations as well as of nonobese and obese control subjects. Interestingly, obese control subjects consumed more frequently meat, white bread, and diet soft drinks than nonobese control subjects. While the increased intake of meat and white bread might be causative for their obesity [20], the enhanced intake of diet soft drinks most likely reflects their attempts to lose body weight [21]. In comparison to the obese control subjects, gastric bypass
Fig. 4 Comparison of dietary habits between gastric bypass patients (gray squares) and gastric banding patients (gray triangles $) .{ }^{(*)} P<0.1, * P<0.05$, $* * P<0.01$

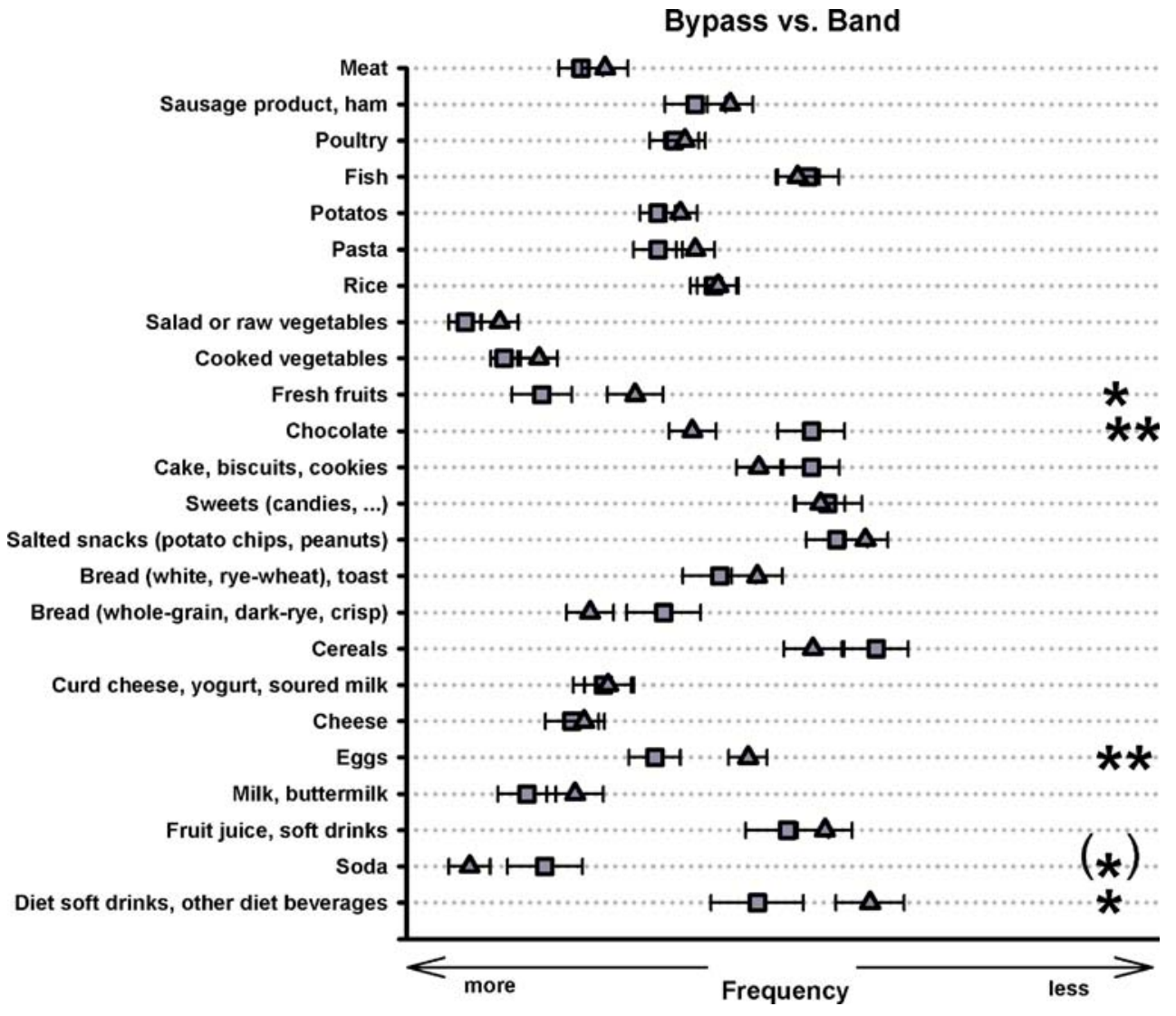


patients showed a more frequent consumption of foods rich in protein such as poultry, fish, and eggs, while they consumed distinctly less fatty sweets such as chocolate, cake, biscuits, and cookies than the obese and nonobese control subjects. Also, gastric bypass patients consumed more cooked vegetables than the subjects of both control groups. Gastric banding patients showed a reduced consumption of pasta, fresh fruits, and white bread while the intake of poultry and fish was enhanced in comparison to the obese control subjects. Direct comparison of the two bariatric groups revealed that gastric bypass patients ingested more frequently fresh fruits, eggs, and diet soft drinks but less frequently chocolate than gastric banding patients. Collectively, our data point to differential changes in dietary habits after gastric bypass vs. gastric banding operations.

Increased intake of high-protein foods such as poultry, fish, and eggs in gastric bypass patients might be best explained by a high compliance with the nutritional advices they received during the dietary counseling sessions. Also, the reduction of chocolate, cake, biscuits, cookies, and of bread might be a result of successful dietary counseling. Alternatively, gastric bypass patients have reduced the consumption of such foods because they have experienced unpleasant symptoms like those of a dumping syndrome or fatty stools after their ingestion and, thus, developed a specific avoiding behavior. However, given that the patients were not asked to provide any explanations for their consumption preferences, the reasons for the observed changes in their intake frequency of specific foods remain to be explored.

In gastric banding patients, the reduced consumption of fresh fruits, pasta, and white bread is likely best explained by the fact that these foods are very difficult to chew good enough for a proper passage through the tight band. By provoking vomiting, these foods might lead to an avoiding behavior as well. However, the increased intake of highprotein foods such as poultry and fish again may reflect a rather high compliance with the dietary advices addressing this issue.

Comparing both bariatric procedures, gastric bypass patients consumed more frequently fresh fruits, eggs, and diet soft drinks and less chocolate than gastric banding patients. Here, it is tempting to conclude that the gastric bypass operation results in a healthier and a more balanced diet than gastric banding. This might be especially true when considering the higher consumption of fresh fruits which are rich in fibers and phytochemicals and, thus, are believed to exert profound cardioprotective and cancer-protective effects [22-24].

Of note, the time interval between the present assessment and the bariatric operation was significantly shorter in the gastric bypass than in the gastric banding patients. Suter et al. [25] previously report on a continuous decrease in food tolerance with increasing time after gastric band implantation, while food tolerance after gastric bypass operations progressively increases over time. Thus, it could well be that changes in dietary habits after different bariatric operations depend on the duration of the postoperative state. If so, this could have significantly contributed to the found differences in food selection after the two different bariatric operations. Also, compliance with dietary advices received before and shortly after the operation will most likely decrease over time which could also have biased our results to some extent.

In conclusion, our results clearly point to distinct changes in dietary habits after bariatric operations, which appear to substantially differ between gastric bypass vs. gastric banding patients. The pattern of changes in food selection after both types of bariatric procedures suggests that patients can, at least in part, follow the dietary advices they receive during dietary counseling. Overall, changes of dietary habits after gastric bypass might be considered more health promoting than dietary changes after gastric banding. However, whether the observed differences in dietary habits translate into clinically relevant differences in outcome measures remains to be elucidated.

\section{References}

1. Demaria EJ. Bariatric surgery for morbid obesity. N Engl J Med. 2007;356:2176-83.

2. Fujioka K. Follow-up of nutritional and metabolic problems after bariatric surgery. Diabetes Care. 2005;28:481-4.

3. Parkes E. Nutritional management of patients after bariatric surgery. Am J Med Sci. 2006;331:207-13.

4. Strader AD, Woods SC. Gastrointestinal hormones and food intake. Gastroenterology 2005;128:175-91.

5. Bobbioni-Harsch E, Huber O, Morel P, et al. Factors influencing energy intake and body weight loss after gastric bypass. Eur J Clin Nutr. 2002;56:551-6.

6. Dias MC, Ribeiro AG, Scabim VM, et al. Dietary intake of female bariatric patients after anti-obesity gastroplasty. Clinics 2006;61:93-8.

7. Warde-Kamar J, Rogers M, Flancbaum L, et al. Calorie intake and meal patterns up to 4 years after Roux-en- $Y$ gastric bypass surgery. Obes Surg. 2004;14:1070-9.

8. Moize V, Geliebter A, Gluck ME, et al. Obese patients have inadequate protein intake related to protein intolerance up to 1 year following Roux-en-Y gastric bypass. Obes Surg. 2003;13:23-8.

9. Trostler N, Mann A, Zilberbush N, et al. Nutrient intake following vertical banded gastroplasty or gastric bypass. Obes Surg. 1995;5:403-10.

10. Trostler N, Mann A, Zilberbush N, et al. Weight loss and food intake 18 months following vertical banded gastroplasty or gastric bypass for severe obesity. Obes Surg. 1995;5:39-51.

11. Naslund I, Jarnmark I, Andersson H. Dietary intake before and after gastric bypass and gastroplasty for morbid obesity in women. Int J Obes. 1988;12:503-13. 
12. Thomas JR, Marcus E. High and low fat food selection with reported frequency intolerance following Roux-en-Y gastric bypass. Obes Surg. 2008;18:282-7.

13. Avinoah E, Ovnat A, Charuzi I. Nutritional status seven years after Roux-en-Y gastric bypass surgery. Surgery 1992;111:137-42.

14. Duran de CC, Dalcanale L, Pajecki D, et al. Calcium intake and metabolic bone disease after eight years of Roux-en-Y gastric bypass. Obes Surg. 2008;18:386-90.

15. Shai I, Henkin Y, Weitzman S, et al. Long-term dietary changes after vertical banded gastroplasty: is the trade-off favorable? Obes Surg. 2002;12:805-11.

16. Silver HJ, Torquati A, Jensen GL, et al. Weight, dietary and physical activity behaviors two years after gastric bypass. Obes Surg. 2006;16:859-64.

17. Brolin RL, Robertson LB, Kenler HA, et al. Weight loss and dietary intake after vertical banded gastroplasty and Roux-en-Y gastric bypass. Ann Surg. 1994;220:782-90.

18. Kenler HA, Brolin RE, Cody RP. Changes in eating behavior after horizontal gastroplasty and Roux-en-Y gastric bypass. Am J Clin Nutr. 1990;52:87-2.
19. Buchwald H, Williams SE. Bariatric surgery worldwide 2003. Obes Surg. 2004;14:1157-64.

20. Newby PK, Muller D, Hallfrisch J, et al. Dietary patterns and changes in body mass index and waist circumference in adults. Am J Clin Nutr. 2003;77:1417-25.

21. Forshee RA, Anderson PA, Storey ML. The role of beverage consumption, physical activity, sedentary behavior, and demographics on body mass index of adolescents. Int J Food Sci Nutr. 2004;55:463-78.

22. Dragsted LO, Krath B, Ravn-Haren G, et al. Biological effects of fruit and vegetables. Proc Nutr Soc. 2006;65:61-7.

23. Dragsted LO, Pedersen A, Hermetter A, et al. The 6-a-day study: effects of fruit and vegetables on markers of oxidative stress and antioxidative defense in healthy nonsmokers. Am J Clin Nutr. 2004;79:1060-72.

24. Beecher GR. Phytonutrients' role in metabolism: effects on resistance to degenerative processes. Nutr Rev. 1999;57:S3-6.

25. Suter M, Calmes JM, Paroz A, et al. A new questionnaire for quick assessment of food tolerance after bariatric surgery. Obes Surg. 2007;17:2-8. 